\title{
Paper-Based RFID-Enabled Wireless Platforms for Sensing Applications
}

Rushi Vyas, Student Member, IEEE, Vasileios Lakafosis, Student Member, IEEE, Amin Rida, Student Member, IEEE, Napol Chaisilwattana, Scott Travis, Jonathan Pan, and Manos M. Tentzeris, Senior Member, IEEE

\begin{abstract}
In this paper, the feasibility of inkjet printing of circuit and microwave structures on paper-based substrates is investigated for the first time in the implementation of a complete low-cost wireless platform for sensors. First, the system-level design of the module including the amplifier characterization were carried out to ensure optimum performance of the sensor modules in the UHF bands used in RF identification communication. These results were then used to design two different antenna structures, which are printed on paper along with their respective circuit layouts using inket-printing technology. Different techniques were investigated for the assembly of circuit components on the silver printed layouts. Finally, wireless link measurements on the assembled prototypes verified the good performance on the wireless and sensing sides.
\end{abstract}

Index Terms-Antenna, assembly, dipole, inkjet printing, microcontroller, monopole, paper, power amplifier (PA), RF identification (RFID), sensor, temperature, transmitter, ultra high frequency (UHF), wireless.

\section{INTRODUCTION}

A S THE demand for low cost, flexible, and power-efficient broadband wireless electronics increases, the materials and integration techniques become more and more critical and face more challenges [1], especially with the ever growing interest for "cognitive intelligence" and wireless applications, married with RF identification (RFID) technologies. This demand is further enhanced by the need for inexpensive, reliable, and durable wireless RFID-enabled sensor nodes that is driven by several applications, such as logistics, aero identification (aero-ID), anticounterfeiting, supply-chain monitoring, space, healthcare, and pharmaceutical, and is regarded as one of the most disruptive technologies to realize truly ubiquitous $a d-h o c$ networks.

In this paper, two completely paper-based RFID-enabled wireless sensor modules operating in the ultra-high-frequency (UHF) are presented for the first time. Paper is considered one of the best organic-substrate candidates for UHF and microwave applications [1]. Paper's biggest advantage is its

Manuscript received August 13, 2008; revised January 13, 2009. First published March 31, 2009; current version published May 06, 2009. This work was supported by the Georgia Electronic Design Center and by the National Science Foundation under Award 0801798.

The authors are with the School of Electrical and Computer Engineering, Georgia Institute of Technology, Atlanta, GA 30332 USA (e-mail: Rushi.Vyas@gatech.edu; vasilis@gatech.edu; arida@gatech.edu; napol@gatech.edu; scorpio@gatech.edu; jpan3@gatech.edu; emmanouil. tentzeris@ece.gatech.edu).

Color versions of one or more of the figures in this paper are available online at http://ieeexplore.ieee.org.

Digital Object Identifier 10.1109/TMTT.2009.2017317

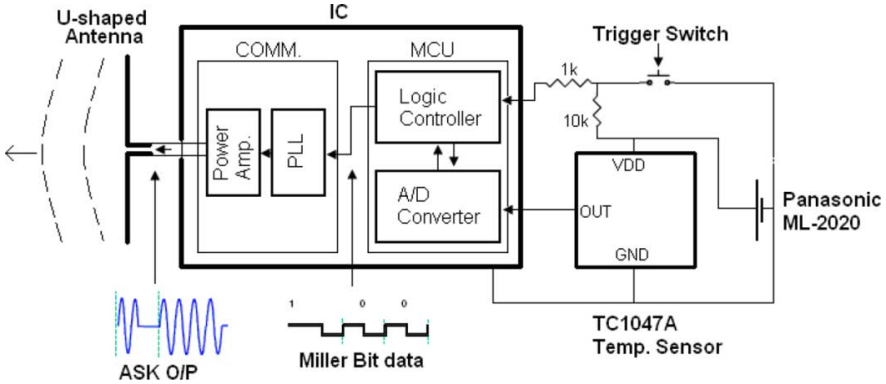

Fig. 1. System-level diagram of the dipole- and monopole-based wireless sensor modules.

high biodegradability with respect to other ceramic substrates such as FR-4, requiring only months to turn into organic matter in landfills. Paper is not only environmentally friendly, but can also undergo large reel-to-reel processing. In terms of mass production and increased demand, paper makes for the lowest cost material made. Paper can also be made to have a low surface profile with an appropriate coating. This is very crucial since fast printing processes, such as direct write methodologies, can be utilized instead of metal etching techniques for metallization on paper. A fast process, like inkjet printing, can be used efficiently to print electronics on/in paper substrates [1].

In this paper, the process to integrate electronics, integrated circuits (ICs), and sensors on paper using inkjet printed technology is presented for the first time. The design process from the system down to the fabrication will be outlined. The constitutive parameters of the paper reported in [2]-[4] will be used to design the microwave structures in the wireless modules. System-level measurements highlighting the feasibility of paper as a suitable low-cost integration platform/package for wireless sensing applications will be demonstrated through wireless link measurements of the sensor information.

\section{Microcontroller AND TransmitTer Design}

To investigate the feasibility of paper as an RF frequency substrate and packaging material, two microcontroller-enabled wireless sensor modules were realized on a photo-paper substrate for the first time. The system-level design for the wireless transmitters can be seen in Fig. 1. At the heart of the system was an 8-bit microcontroller unit (MCU) integrated with a UHF amplitude shift keying (ASK) transmitter in a single IC chip. Having an integrated MCU with a transmitter offered the versatility of customizing the system for use with any kind of analog or even digital sensor, whose data could be sent out wirelessly with a maximum user-defined data rate. The data transmission was to be carried out at the unlicensed industrial, scientific, and 
medical frequency band around $900 \mathrm{MHz}$ in a way similar to RFID tags that use EPCglobal's GEN-2 protocol [5]. The first wireless sensor module prototype using a dipole antenna was printed on a 2-D (single layer) paper module, and the second prototype using a monopole antenna was printed on a 3-D (multilayer) paper module. The full design process will be outlined in this section.

\section{A. MCU Operation}

An integrated 8-bit MCU was used as the primary controlling mechanism of the wireless sensor module. The MCU used a high-performance reduced instruction set computing (RISC) architecture, with its own internal 4-MHz oscillator that was calibrated to within $1 \%$. The MCU was programmed using an assembly code, which offers the best control over the timing of the device instructions, and hence, the I/O ports of the MCU.

The MCU was programmed to operate in three different modes of operation, which could be selected by the user with an external trigger switch (push button) shown in the system-level diagram in Fig. 1. In mode 1 (UNMOD), the MCU was programmed to turn on the COMM module, and have it send out an unmodulated signal at the design frequency around $900 \mathrm{MHz}$. In mode 2 (SENSE), the MCU was programmed to read temperature from an external temperature sensor and have the COMM module send out the sensor data using an encoded ASK modulated signal. In mode 3 , the MCU was programmed to disable the COMM module and go into sleep mode (SLEEP), an extremely low power state $(\cong 1.8 \mu \mathrm{W})$, in order to conserve power [6], [10]. In the UNMOD mode, during the full unmodulated signal transmission, the power consumption was measured to be $36 \mathrm{~mW}$, which would enable the wireless sensor module system to operate for a maximum period of $3.75 \mathrm{~h}$ with a 3-V battery capable of supplying $45 \mathrm{~mA} / \mathrm{h} \mathrm{[7].}$

The MCU contained an integrated 10-bit analog-to-digital converter that was setup to sample a Microchip TC1047A analog temperature sensor in the SENSE mode. All ten bits of the A/D converter were utilized for maximum sensing resolution. The temperature sensor was sampled four times during one read operation to average out variations in the sampled sensor output in between its sensor readings. The sampled analog data were then converted to their respective 10-bit digital form and stored in the MCU's program memory, where they were averaged. In the assembly code, the averaging was performed by summing all four of the stored digital sensor data and performing a bit shift operation on the sum twice to perform a divide by 4 operation.

The averaged sensor data in its digital form was then bit encoded using a complete two sub-carrier cycle Miller bit encoding, as shown in Fig. 2. Miller bit encoding is one of the most common symbol types used in RFID's GEN-2 protocol [6], [8]. In it, 2- or 3-bit transitions occur in between a "1" or " 0 " symbol, respectively, and no two consecutive 0 or 1 bits can be identical, as can be seen in the sequence shown in Fig. 4 . This makes it possible to communicate the bits asynchronously, making it easier to decode them on the receiver side, while conserving bandwidth. As a final step, the bit-encoded sensor data was then transmitted wirelessly by using the MCU to control the integrated communication module (COMM).

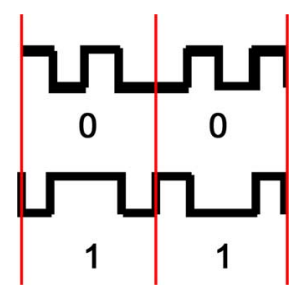

Fig. 2. Two sub-carrier cycles Miller bit encoding.

\section{B. Communication Module (COMM) Operation}

The transmit frequency was generated with the help of an external crystal oscillator fed into the communication module (COMM) of the IC in Fig. 1, which comprised of a phase-locked loop (PLL) module with a power amplifier (PA) at the output of the wireless transmitter. The COMM module, when enabled by the MCU, takes in the input or reference frequency generated by the RF crystal oscillator and generates an output signal locked in at 32 times the reference frequency. The $\mathrm{MCU}$ was programmed to produce a clock delay of $600 \mathrm{~ms}$ after enabling the integrated COMM module, which is roughly equal to the startup time of the crystal oscillator and the acquisition time of the PLL. The acquisition time is the amount of time taken by the PLL to lock at which point the output of the phase/freq. detector and the voltage-controlled oscillator block in Fig. 3 below are $0 \mathrm{~A}$ and $0 \mathrm{~V}$, respectively [9]. The PLL lock occurs only when its output frequency is equal to 32 times the reference frequency of the RF crystal oscillator [9].

The MCU was programmed to control the wireless communication by modulating the PA in the integrated communication module (COMM) at calculated intervals, thereby giving an ASK modulated signal at the output of the PA with the required data rate [6]. The modulation by the MCU was carried out in the same sequence as the bit encoded sensor data that was sampled and averaged during the previously described SENSE mode. The output data rate was generated by using the internal clock of the MCU to toggle the RF PA. With a 4-MHz internal clock, the time to execute one instruction was four times the clock period, which was $1 \mathrm{~ms}$ by the MCU [10]. This clock delay was used to control the time duration between the enabling and disabling of the PA thereby controlling the modulated RF output waveform and by it the data rate shown in Fig. 4. The number of program instructions $n$ required by the MCU to produce the correct delay between the toggling of the PA that would generate a desired data rate (DataRate) can be determined from (1) as follows:

$$
\text { DataRate }=\left(n \times x \times \frac{1}{f_{\mathrm{MCU}-\mathrm{CLK}}}\right)^{-1}
$$

where $x$ is the cycles-per-clock delay in implementing one instruction by the program counter, which was set to the lowest allowable value of 4 , as permitted by the MCU architecture in order to give the fastest data rate. The parameter $f_{\mathrm{MCU}-\mathrm{CLK}}$ in (1) is the clock frequency of the MCU. To minimize the number of the external components, it was decided to use the integrated Colpitts oscillator internal to the MCU, which had a maximum clock frequency of $4 \mathrm{MHz}$ for both monopole- and dipole-based designs. Fig. 4 shows the MCU generated signals at the COMM 


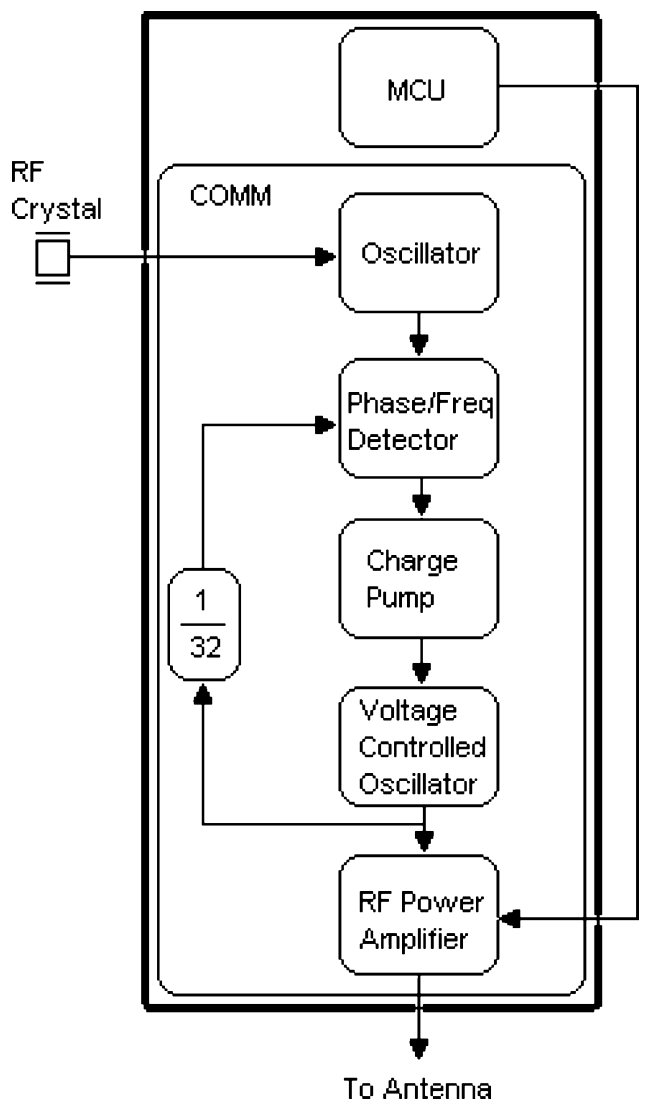

Fig. 3. System-level diagram of wireless transmitter.

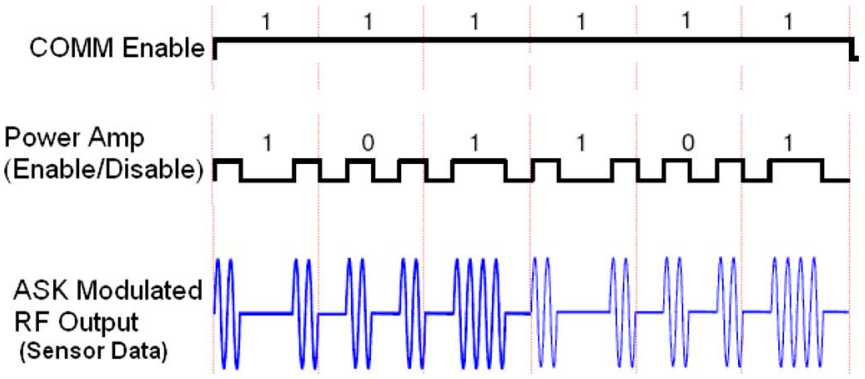

Fig. 4. COMM module inputs and output (time axis not per scale).

enable and PA enable inputs of the COMM module. The sinusoidal waveform in Fig. 4 is the final ASK modulated RF output of the PA, which is also the sensor data that is fed into the antenna.

Two sub-carrier cycle Miller bit encoded signals are transmitted at data rates in excess of $5 \mathrm{~kb} / \mathrm{s}$ from tags to reader as per RFID UHF Gen-2 protocols [8]. Based on (1), any value of $n$ less than 200 should yield a data rate of greater than $5 \mathrm{~kb} / \mathrm{s}$ for an MCU clock frequency of $4 \mathrm{MHz}$. For the module prototypes presented in this paper, the value of $n$ that was coded in the MCU firmware was 146, which yielded a data rate of $6.8 \mathrm{~kb} / \mathrm{s}$, as was verified by the wireless link measurement shown in Fig. 5. The data rate is the reciprocal of the measured bit duration shown in Fig. 5.

To verify the correct operation of the MCU firmware and its interoperability with the COMM module, a prototype of the entire system was implemented on a copper-clad Duroid board,

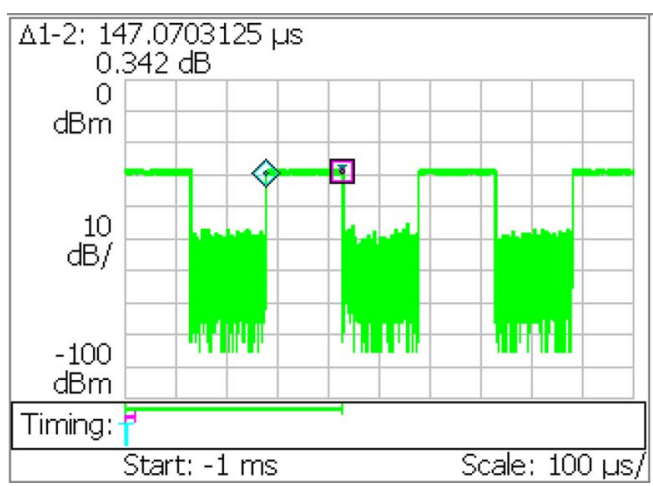

Fig. 5. RTSA measured ASK modulated signal with 50\% duty cycle (power versus time).

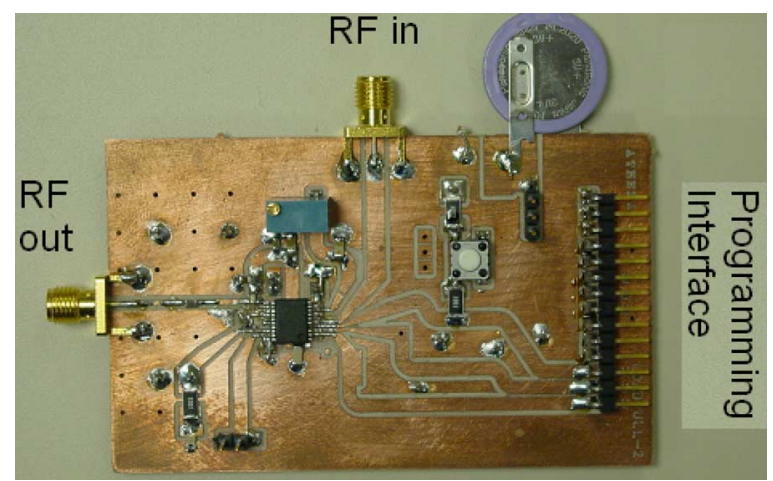

Fig. 6. Prototying board for load-pull analysis and in-circuit programming [12].

as shown in Fig. 6. Interfaces were also provided to the IC on the prototype board to enable in-circuit programming and debugging of the MCU. The PA output was connected to a printed circuit board (PCB)-mounted subminiature A (SMA) connector through a coplanar waveguide with a grond plane (CPW-G) feed for the RF baud rate and PA characterization measurements described in the following section.

\section{Amplifier (PA) Characterization}

The range of the wireless transfer is directly proportional to the amount of power transferred from the amplifier in the transmitter front end to the antenna. Any impedance mismatch between the two can lead to the reflection of a part of the power intended to radiate out of the antenna, thereby minimizing the range. Instead of designing the antenna to a $50-\Omega$ match, it was decided to design it to the optimum impedance looking out of the amplifier (PA) in the transmitter. Doing so would eliminate the need for a matching network, minimizing series losses, as well as the number of components used in the design, thus leading to a more compact design.

To determine the optimum load impedance looking out of the PA in the transmitter, a load-pull analysis was performed on it at three different frequencies of 860, 904.4, and $925 \mathrm{MHz}$ [11], all of which fall within the unlicensed UHF frequency bands in the U.S. and Europe used for RFID communication [5]. The analysis was carried out for a fixed-bias point for which the PA output was biased at the power supply voltage of $3 \mathrm{~V}$ with an $\mathrm{RF}$ choke in between to maximize drain collector efficiency for 

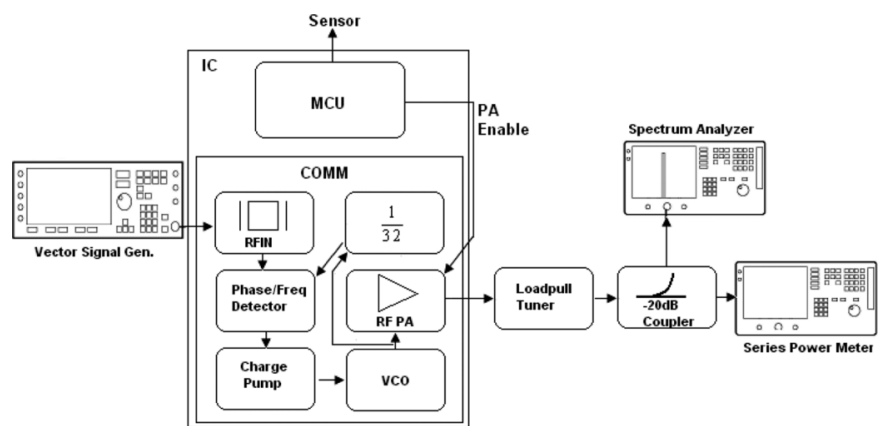

Fig. 7. Measurement setup for load-pull characterization of integrated PA.

TABLE I

LOAD-PULL RESULTS

\begin{tabular}{|c|c|c|c|}
\hline & $860 \mathrm{MHz}$ & $904.4 \mathrm{MHz}$ & $925 \mathrm{MHz}$ \\
\hline $\mathrm{Z}_{\text {tuner }}$ (ohms) & $18.63+\mathrm{j} 27.4$ & $75.5+\mathrm{j} 103.6$ & $157.7-\mathrm{j} 15.8$ \\
\hline $\mathrm{Z}_{\text {bias }}$ (ohms) & $12.83+\mathrm{j} 290.7$ & $15.43+\mathrm{j} 299.5$ & $15.45+\mathrm{j} 302.2$ \\
\hline $\mathrm{Z}_{\mathrm{L}-\text { opt }}(\mathrm{ohms})$ & $117.5+\mathrm{j} 137.1$ & $60.1-\mathrm{j} 73.51$ & $35.86-\mathrm{j} 15.87$ \\
\hline $\mathrm{Z}_{\mathrm{X}}$ (ohms) & $275.9+\mathrm{j} 61.67$ & $37.31-\mathrm{j} 65.96$ & $32.1-\mathrm{j} 18.88$ \\
\hline $\begin{array}{c}\text { Output Power } \\
\text { (Measured) } \\
\text { (dBm) }\end{array}$ & 6.1 & 7.43 & 7.72 \\
\hline $\begin{array}{c}\text { Output Power } \\
\text { (Manufacturer) } \\
(\mathrm{dBm})\end{array}$ & 7.5 & 7.5 & 7.5 \\
\hline
\end{tabular}

class A operation suitable for ASK modulation [11]. The setup used for this measurement is shown in Fig. 7. For this measurement, the test module, built on Duroid and shown in Fig. 6, was used.

For the load-pull measurements, the microcontroller was programmed to operate in the UNMOD mode during which it would continuously enable the RF PA, which would send a constant unmodulated sinusoidal signal at the transmitter output. The load-pull tuner was tuned to different impedances to locate its impedance value $\left(Z_{\text {tuner }}\right)$ at which the PA gave out maximum power [11], which was read off the series power meter (see Fig. 6). The loss in the measurement setup, i.e., the $\mathrm{RF}$ tuner, the $-20-\mathrm{dB}$ coupler, and the cables, were measured separately at each one of the three measurement frequencies and added to the power output measured with the power meter to get the optimum output power shown in Table I. An Agilent E4438C vector signal generator (VSG) was used to generate the input RF signal, while the output power was measured using the Agilent E4419B rpm power meter. A portion of the output power was coupled to an Agilent E4404B spectrum analyzer to ensure that the power meter was measuring the output power at the correct output RF frequency. The load-pull tuner used was a Maury model 1643-N.

The effects of the SMA connector, coplanar waveguide (CPW) feed, the series capacitor, and bias circuitry were deembedded at various points along the output of the circuit to determine the optimum impedance referenced at the IC output $\left(Z_{\mathrm{L}-\mathrm{opt}}\right)$ after accounting for the impedance of the bias circuit.

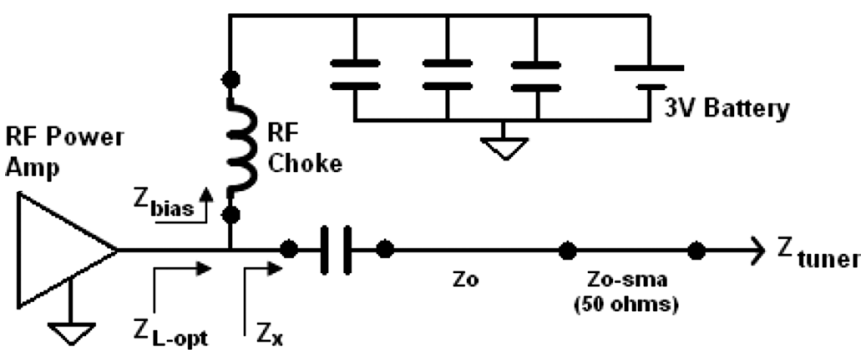

Fig. 8. Equivalent circuit looking out of the RF PA.

The impedance of the bias circuit $\left(Z_{\mathrm{bias}}\right)$ was determined by measuring the $S$-parameters of the RF choke and bias coupling capacitors from which their respective impedances were determined at $860,904.4$, and $925 \mathrm{MHz}$ that were then modeled in the bias circuitry using Agilent's Advanced Design System (ADS) software. The equivalent circuit looking out of the PA on the prototyping board that had to be deembedded for the load-pull test setup is shown in Fig. 8. The results of the load-pull analysis are shown in Table I.

\section{ANTENNA DESIGN AND CiRCUIT INTEGRATION}

The output impedances of the PA determined during the load-pull characterization were then used to design power-optimized dipole and monopole antennas for the wireless transmitters. The antennas were designed to resonate close to 904.4 MHz with the hopes of achieving a return loss bandwidth that would cover the extreme frequencies of 860 and $925 \mathrm{MHz}$ as well. The antennas were inkjet printed on the paper substrates along with the circuit itself.

\section{A. Single-Layer Dipole Antenna}

Half-wavelength dipole antennas are among the most commonly used antennas for RFID applications. They can be folded and tapered for more compact size and wider bandwidth [13], [14]. In addition, half-wavelength dipole antennas have a radiation resistance of $75 \Omega$ [15], which is close to the real part of the optimum load impedance $\left(Z_{\mathrm{L}-\mathrm{opt}}\right)$ looking out of the PA at 904.4 MHz listed in Table I making for an easier impedance match. For the first wireless sensor module prototype, a tapering U-shaped half wavelength dipole structure described in [12] and [16] was chosen for the antenna. The circuit layout was to be placed in the space within the U-shape of the antenna to make the size of the complete module more compact [3], [16].

The antenna was to be printed on a single layer of a paper substrate along with the circuit layout using an inket-printing technique [6], [16]-[19]. The biggest challenge on using a dipole antenna this way was integrating and sufficiently isolating it from the circuit of the sensor module within its U-shape, as shown in Fig. 9. The close vicinity of the antenna with respect to the circuit would alter its mutual impedance, which could cause an impedance mismatch with the PA, thereby reducing the amount of power radiated along with the transmitted range [15]. To ensure an impedance match, the antenna was optimized so that the impedance looking out of the PA would be close to its optimum value $\left(Z_{\mathrm{L}-\mathrm{opt}}\right)$ at $904.4 \mathrm{MHz}(60.1-j 73.51 \Omega)$ with 


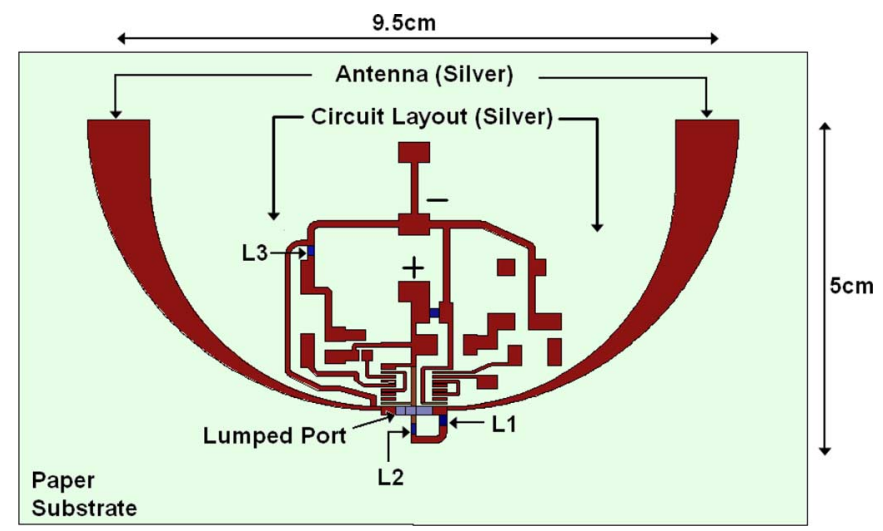

Fig. 9. Dipole-based module topology.

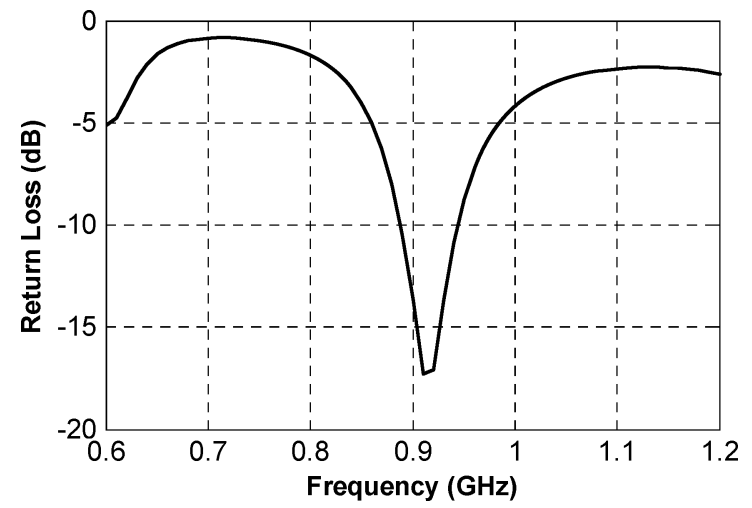

Fig. 10. Simulated return loss of the dipole antenna connected to the circuit.

the sensor module circuitry connected within the space between the antenna arms.

In addition, RF chokes (L1, L2) were used at several points in the circuit to minimize RF signals, meant to travel between the antenna and PA, from creeping into the positive power supply trace of the battery that was used to bias the PA (antenna terminals) and power up the MCU. RF chokes could not, however, be placed between the negative terminal of the antenna (also the PA ground) and the negative power supply of the battery because doing so would reduce the gain of the single-ended PA and alter the optimum load impedance determined in Table I [11]. An RF choke (L3) was placed between the RF crystal and the negative power supply of the battery to isolate it from RF signals from the antenna.

The entire structure of the module including the antenna and the circuit layout was built using Ansoft's HFSS 3-D EM solver, which was also used to optimize the antenna. Lumped resistor/ inductor/capacitor (RLC) boundaries with values equal to the measured values of inductors L1, L2, and L3 at $904.4 \mathrm{MHz}$ were used to simulate the RF chokes in the circuit, as shown in Fig. 9. A lumped port was used as the RF power source in place of the PA to excite the antenna. The final dipole antenna design along with the circuit in between had a dimension of $9.5 \times 5 \mathrm{sq} . \mathrm{cm}$.

The simulated return loss or $S_{11}$ for the antenna with respect to the PA's optimum load impedance $(60.1-j 73.51 \Omega$ ) is shown in Fig. 10, and shows a good impedance match at the design frequency of $904.4 \mathrm{MHz}$ with a -10 -dB bandwidth of $60 \mathrm{MHz}$. The simulated and measured radiation pattern are
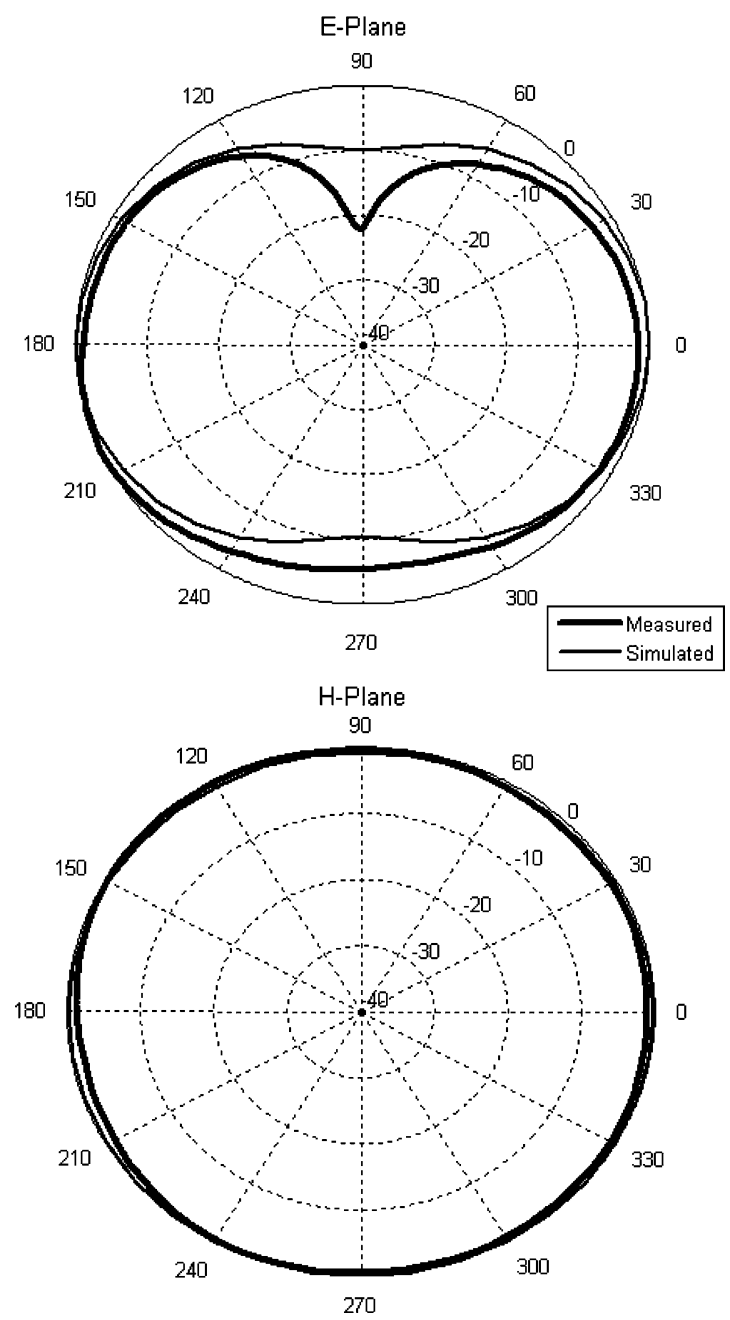

Fig. 11. Normalized 2-D far-field radiation plots of simulation and chamber measurement of the dipole-based printed sensor module.

shown in Fig. 11 and show reasonably good agreement. A maximum simulated directivity of $1.54 \mathrm{~dB}$ was achieved.

\section{B. Double-Layer Monopole Antenna}

Many of the drawbacks with the dipole-based module can be eliminated by using a monopole-based structure. The monopole uses its ground planes as a radiating surface, which can also be used to shield any circuitry behind it. The monopole antenna also does not require a differentially fed input signal like the dipole, which was ideal for the PA since its output was single ended.

The circuit for the monopole was laid out on two layers, which helped minimize the size of the circuit topology by avoiding the long power supply traces that had to be used on the single-layer dipole-based module. The top layer contained the printed antenna and most of the circuit components for the module. The bottom layer contained an Li-ion cell and the power supply traces, which were routed to the top layer through drilled vias.

The monopole antenna had a planar CPW-G fed wideband structure with a rectangular radiator to achieve a more compact and wideband design that could be easily printed [20]-[22]. A CPW-G on the top and bottom layers is extremely suitable at 


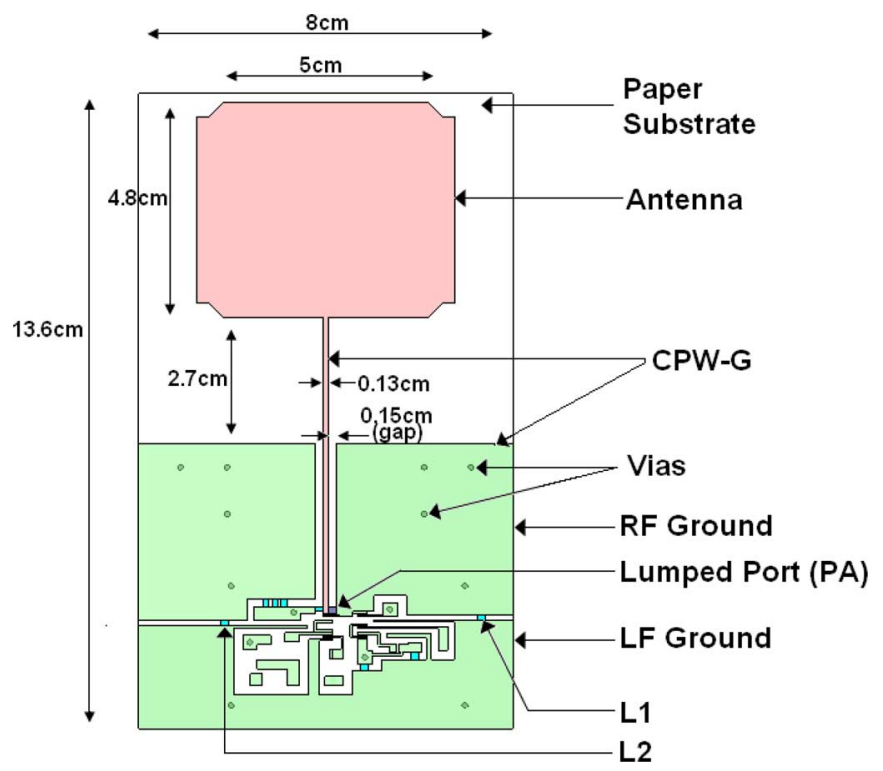

Fig. 12. Monopole-based module topology.

shielding the antenna and the sensor data bus from interfering noise that may have coupled into the shared power supply traces in the bottom layer and also due to the digital switching within the MCU on the top layer [23]. In addition, the CPW-G feed line could also allow a matching network to be implemented between the PA and antenna in the event of a possible mismatch between the two. The monopole-based sensor module topology is shown in Fig. 12.

The entire topology, shown in Fig. 12, was also simulated using Ansoft's High Frequency Structure Simulator (HFSS) 3-D electromagnetic (EM) tool. Multipoint grounds (RF and LF) were used for this design for better isolation between the digital switching occurring in the MCU and RF transmission [23]. RF chokes (L1 and L2) simulated as lumped RLC boundaries were once again used to isolate the two grounds, as shown in Fig. 12. A lumped port was used as the RF source to replicate the PA for the simulation. The antenna was matched to an impedance of $60.1-j 73.51 \Omega\left(Z_{\mathrm{L}-\mathrm{opt}}\right)$, which is the reference at the PA output shown in Fig. 8 at the design frequency of 904.4 MHz. The simulated return loss for the entire structure showed good wideband resonance of about $220 \mathrm{MHz}$ around the design frequency of $904.4 \mathrm{MHz}$, as shown in Fig. 13. The maximum simulated directivity obtained was $2.6 \mathrm{~dB}$. The measured and simulated radiation patterns are shown in Fig. 14.

\section{FABRICATION AND ASSEMBLY}

Once the system topology and antennas had been designed, the overall performance of the prototype board shown in Fig. 7 was verified. The complete designs were then reproduced on paper using inket-printing technology. The antennas and the circuit layouts were printed and cured on paper using silver ink and the complete wireless sensor system comprising the IC (MCU+ transmitter), its discrete passive components, the temperature sensor, and an Li-ion cell for "standalone" (autonomous) operation were assembled on it. The fabrication/assembly process is outlined in this section.

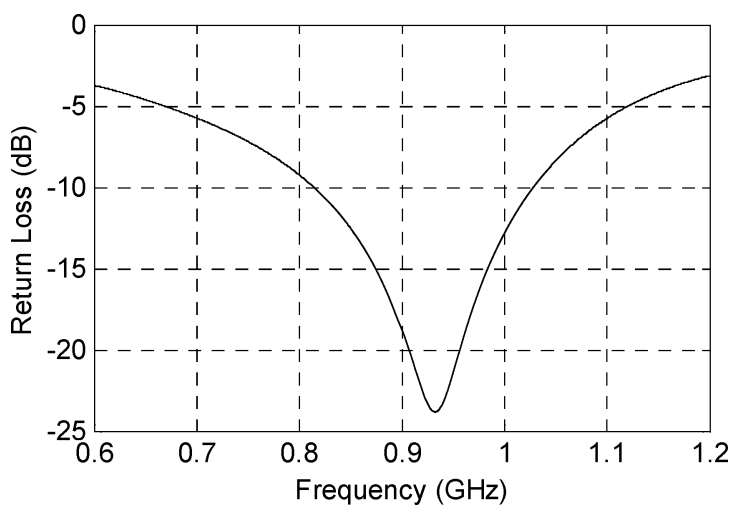

Fig. 13. Simulated return loss of the monopole antenna connected to the circuit.
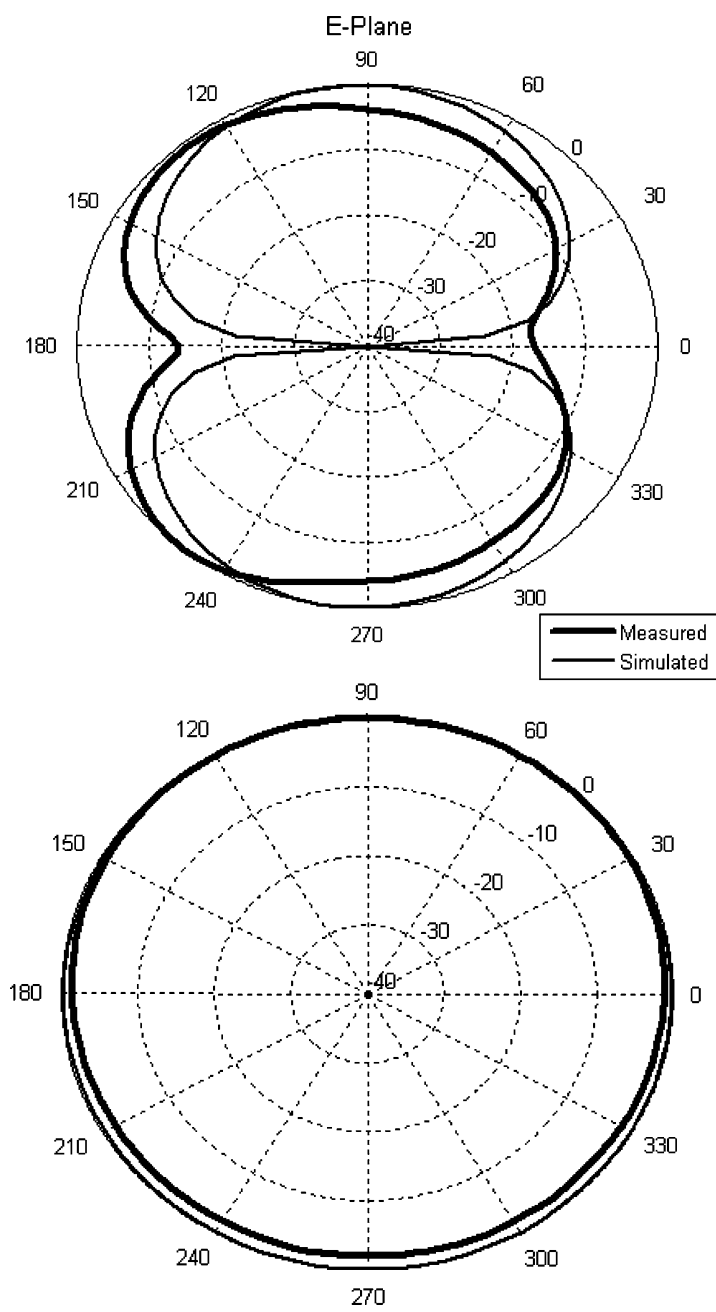

Fig. 14. Normalized 2-D far-field radiation plots of simulation and chamber measurement of the monopole-based printed sensor module.

\section{A. Inkjet-Printing Technique}

The proposed circuits for the wireless sensor module including the antennas and circuit layouts were designed and simulated using HFSS software, and fabricated using an inket-printing technique. In inkjet printing, the conductive silver ink droplets are directly sprayed from the inkjet nozzle onto the paper-based substrate, thus eliminating the waste 


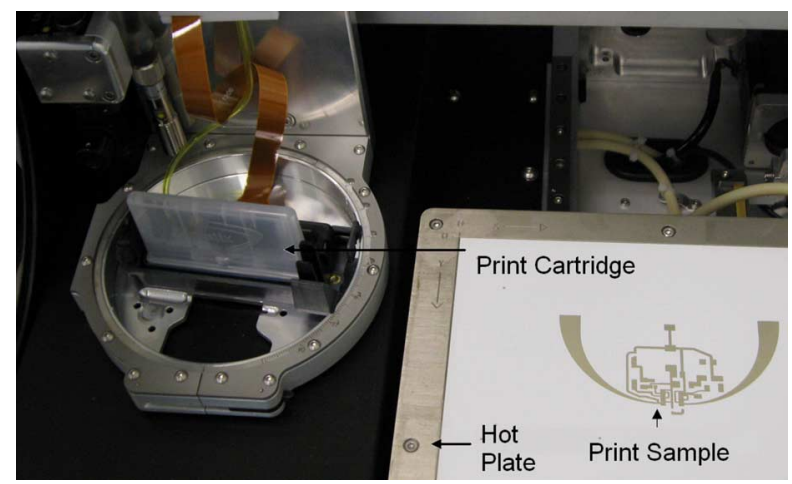

Fig. 15. Dimatix printing chamber.

generated using traditional photolithography techniques, which etch out unwanted metal at the end of the process [1].

A Dimatix DMP-2800 inkjet printer (printer) was used to carry out the inkjet printing. The conductor consisted of a conductive ink containing silver nanoparticles, which was sprayed onto a paper-based substrate using the inkjet printer to ensure good metal conductivity [1]. To ensure maximum conductivity and antenna efficiency, the entire circuit was printed over with 12 layers of silver ink resulting in a conductor thickness of $12 \mu \mathrm{m}$ [14].

The substrate was sintered continuously during the fabrication process at $60{ }^{\circ} \mathrm{C}$ using the hot base plate in the printing chamber of the printer shown in Fig. 15. The sintering process removed excess solvent and material impurities from the depositions and increased bonding between the silver ink and paper [24].

The layers were printed using two different types of piezoelectric inket-printing cartridges. The high precision cartridge that sprayed 1-pL droplets was used to print finer areas in the circuit layout such as the IC traces and the PA interface to the antenna $(150 \mu \mathrm{m})$. The 10-pL cartridge was used to print larger areas on the antenna and the RF ground planes/traces in both the monopole and dipole circuits to increase conductivity. This was done to minimize the gap between adjacently sprayed silver particles enabling a larger overlap during the annealing process and ensuring better conductivity of the printed structure. A close-up picture of the overlap between the printed ink layers using the 10 -pL cartridge is shown in Fig. 16. The printed structures were then cured in an industrial oven at $100{ }^{\circ} \mathrm{C}$ for $10 \mathrm{~h}$. This was done with the intent to increase the conductivity of the printed structures toward the higher end of the conductivity range achievable with this process, which is between $0.4 \sim 2.5 \times 10^{7} \mathrm{~S} / \mathrm{m}$ [2]. The entire printing process was repeated to print the individual layers used in the dipole-based (one layer) and monopole-based designs (two layers).

\section{B. Circuit Assembly}

Next, the wireless sensor modules were assembled on the printed circuit. The assembly process on silver pads proved to be the most challenging aspect in the design process. Given the low temperature tolerance of paper, the electronic components used, and the relative weaker adhesion of printed silver pads on paper, soldering had to be ruled out. Multiple assembly methods were

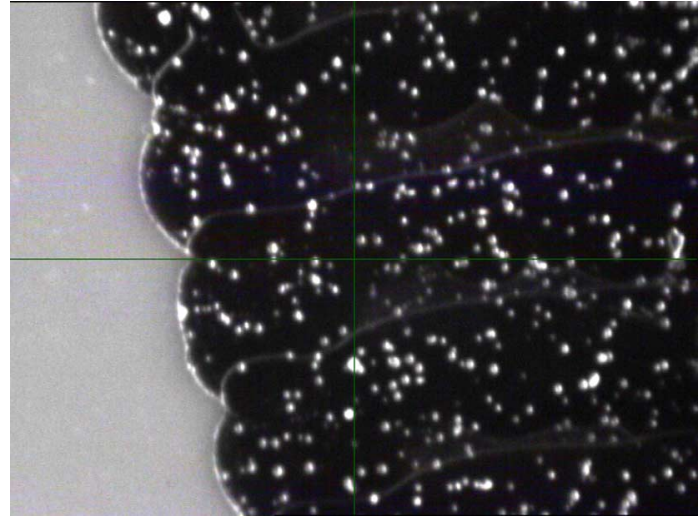

Fig. 16. Fiducial camera image showing closeup of silver ink layers printed using 10-pL inkjet cartridge (zoom factor: $\times 100$ ).

experimented in order to find a reliable alternative for mounting components which include silver epoxies and conductive tapes.

The procedure for mounting components on the printed circuits was identical for both monopole- and dipole-based modules. The wireless sensor module using the monopole, due to its two-layer design, required additional steps to the assembly process. For the monopole-based module, the bottom layer containing the printed ground plane and the positive power supply trace was laminated to the top layer containing the printed circuit, monopole antenna, and signal feed line prior to the circuit assembly process. Roughly $5 \mathrm{lb} / \mathrm{sq}$. in of pressure was applied on the two layers for $30 \mathrm{~min}$ until the bonding adhesive dried sufficiently.

Via-holes were then drilled through the laminated structure using a $400-\mu \mathrm{m}$ microdrill bit manufactured by LPKF A.G., Garbsen, Germany. Fiducial marks printed along the borders were used to ensure that the top and bottom layers of the monopole-based wireless sensor module were aligned properly. The drilled via-holes were then filled using silver epoxy. A small area of the adhesive was left to spill over from the via-holes across the top and bottom silver traces to ensure connection. The structure was then cured at $120{ }^{\circ} \mathrm{C}$ for $20 \mathrm{~min}$.

Two different silver epoxies were investigated for making conductive electrical contacts between the circuit components and the printed silver traces given the unfeasibility of using solder on printed silver. The first type had a thinner texture prior to curing, which made it conducive to applying onto smaller circuit points. It could be easily applied to the finer interfaces between the components and printed silver traces. However, given its inability to hold components in place, nonconductive adhesive had to be applied first under the component terminals, as shown in Fig. 17.

By comparison, the second type of epoxy used, which proved to be a better choice, had a denser texture providing a secure grip of the components prior to curing although it was harder to apply on finer areas in the circuit. A comparison between the two epoxy textures can be seen in Fig. 18. Both of the epoxies, after application, had to be cured in an oven to harden its textures and improve conductivity. The denser epoxy also has a much higher thermal conductivity of $12.7 \mathrm{~W} / \mathrm{mK}$ compared to $2 \mathrm{~W} / \mathrm{mK}$ for the thinner epoxy, resulting in a lower curing time, 


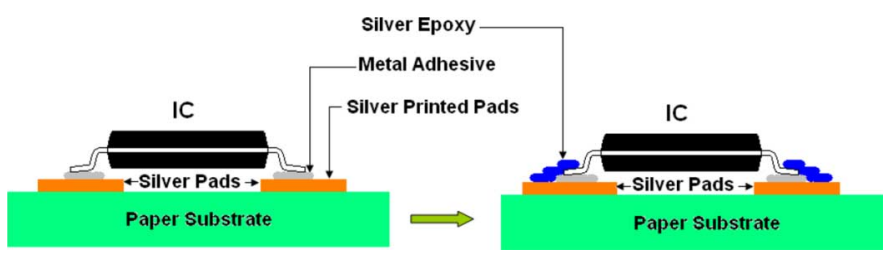

Fig. 17. Assembly process for components on printed silver pads using the Epo-Tek silver epoxy.

\begin{tabular}{|l|l|l|}
\hline & $\begin{array}{l}\text { Epoxy with thinner } \\
\text { texture }\end{array}$ & $\begin{array}{l}\text { Epoxy with } \\
\text { denser texture }\end{array}$ \\
\hline Before Cure & & \\
\hline After Cure & & \\
& & \\
\hline
\end{tabular}

Fig. 18. Silver epoxy textures before and after curing.

TABLE II

Curing Order, Temperature, AND Time Used for Assembly Process

\begin{tabular}{|l|l|l|l|}
\hline Order & Components & $\begin{array}{l}\text { Curing } \\
\text { Temperature } \\
\text { (Celsius) }\end{array}$ & $\begin{array}{l}\text { Curing Time } \\
\text { (minutes) }\end{array}$ \\
\hline 1 & TC1047 Temp Sensor & 120 & 15 \\
\hline 2 & $\begin{array}{l}\text { IC, Inductors, Capacitors, } \\
\text { Resistors }\end{array}$ & 100 & 45 \\
\hline 3 & Crystal Oscillator & 80 & 90 \\
\hline 4 & ML 2020 Panasonic Cell & 60 & 240 \\
\hline
\end{tabular}

which was more conducive to the electrical components with lower temperature tolerances.

The entire assembly process had to be carried out in a multistep process [12]. This was because the conductivity of the silver epoxy had a direct co-relation to its curing time and temperature, which contradicted with the limited temperature tolerance of the components used in the wireless transmitter. Due to this, the assembly of components was done in the order shown in Table II [12]. The curing time and temperature are also shown in Table II.

\section{System-LeVel Performance}

Wireless link measurements were then performed with the assembled modules to verify their system-level performance. The wireless link measurements were carried out by placing the modules at different temperatures within an oven or refrigerator, and triggering them to transmit in the SENSE mode. On the receiver side, the ASK modulated wireless sensor information was captured using a wideband AN-400 RFID reader antenna connected to a Tektronix RSA 3408A real time spectrum analyzer (RTSA). The transmitter was kept at a distance greater than its

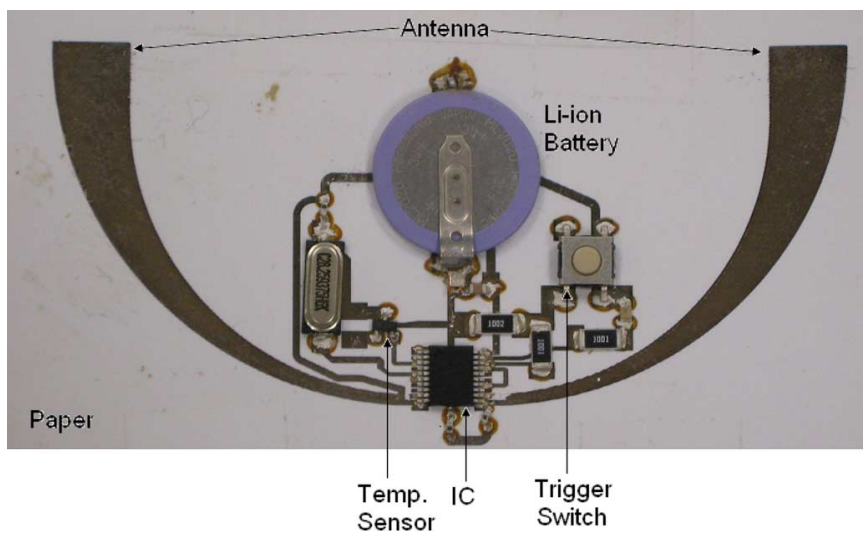

Fig. 19. Dipole-based wireless sensor module on paper substrate using inketprinting technology.

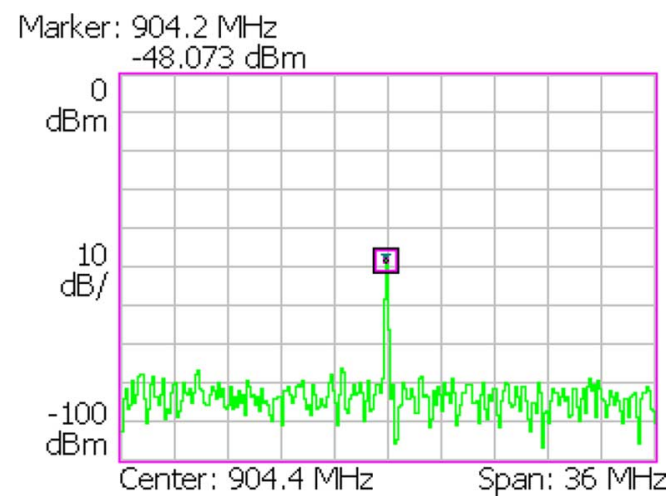

Fig. 20. RTSA measured ASK modulated signal for the dipole-based module from a distance of $4.26 \mathrm{~m}$ (power versus frequency).

far-field boundary from the receiver. The far-field boundary of the dipole and monopole-based modules were determined to be 3.8 and $5.4 \mathrm{~cm}$ using Fraunhofer's far-field equation shown in (2) as follows [15]:

$$
R=\frac{2 D^{2}}{\lambda}
$$

In (2), $R$ is the distance from the transmitter at which its far field region starts, $D$ is the largest dimension of the antenna, and $\lambda$ is the free-space wavelength at the transmit frequency of $904.4 \mathrm{MHz}$. The wireless sensor data captured by the RTSA were decoded and compared to the temperature obtained from a commercial noncontact infrared (IR) thermometer. The results have been summarized in this section.

\section{A. Dipole-Based Wireless Sensor Module}

The assembled dipole-based wireless sensor module can be seen in Fig. 19. The transmitted signal measured by the RTSA from a distance of $4.26 \mathrm{~m}$ can be seen in Fig. 20, and was observed to be $-48.07 \mathrm{dBm}$. The transmission obtained with the dipole-based sensor module presented in this paper was observed to be significantly better than the first prototype designed in [12] whose transmission from a distance of $0.3 \mathrm{~m}$ was only $-69 \mathrm{dBm}$, shown in Fig. 21, and which was designed without accounting for the circuit and printing effects on the antenna. 


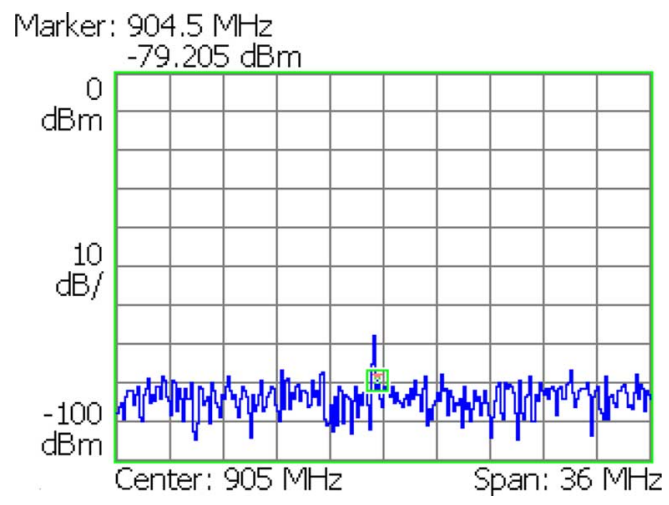

Fig. 21. RTSA measured ASK modulated signal for the dipole-based module from a distance of $0.3 \mathrm{~m}$ from [12] and [25] (power versus frequency).

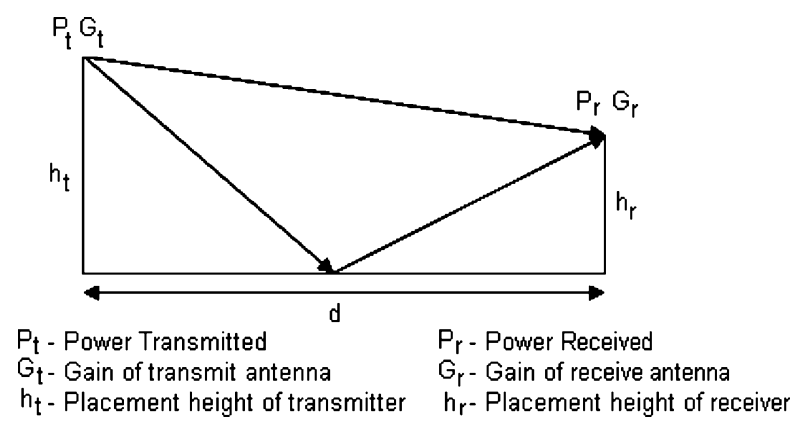

Fig. 22. Link geometry for wireless link analysis [26].

Based on the transmission measurements carried out under laboratory conditions, a wireless link budget analysis was performed on the dipole-based sensor module from which its expected range was determined. The wireless link geometry used can be seen in Fig. 22. For the measurement setup used, the placement heights of the transmitter and receiver used were 1.17 $\left(h_{t}\right)$ and $0.97\left(h_{r}\right) \mathrm{m}$, respectively. The transmitter was placed at a distance of $4.26 \mathrm{~m}(d)$ from the receiver.

For the measurement setup used, the maximum radius of the first Fresnel zone $r$ obtained at a midpoint between the transmitter (Tx) and receiver (Rx) was determined using (3) [26], where $\lambda$ is the wavelength of the transmitted signal. The lengths $d_{t}$ and $d_{r}$ in (3) represent the distance of the Fresnel zone cross section under consideration from the transmitter and receiver, respectively, as shown in Fig. 23

$$
r=\sqrt{\frac{\lambda}{\left(\frac{1}{d_{t}}+\frac{1}{d_{r}}\right)}} .
$$

The first Fresnel zone radius for the dipole-based module $(r)$ was determined to be approximately $0.5945 \mathrm{~m}$, which is lower than the heights of the transmitter and receiver. Based on this assessment, the requirement of the clearance of the first Fresnel zone was satisfied and, due to which, the free-space loss model was the most suited for determining the wireless link budget for the dipole-based module [27].

Using the free-space loss model for the measurement setup shown in Fig. 22, the maximum effective isotropic radiated

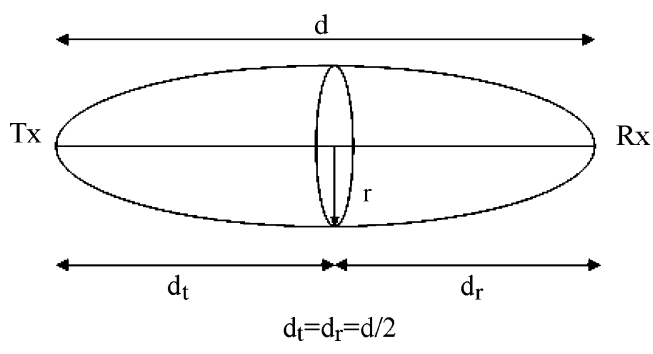

Fig. 23. Link geometry for wireless link analysis [26].

power (EIRP) from a transmitter can be determined using (4). On the receive side, the AN-400 Reader antenna had a gain $\left(G_{r}\right)$ of $6 \mathrm{dBi}$ [28], which received a signal strength of $-48.073 \mathrm{dBm}$ $\left(P_{r}\right)$ that was measured by the RTSA (see Fig. 20). Using (4), the maximum amount of EIRP that was given out by the dipole-based sensor module was determined to be $-9.9 \mathrm{dBm}$

$$
\mathrm{EIRP}=P_{r}-G_{r}+20 \log (4 \pi d)-20 \log (\lambda) .
$$

The maximum range of the transmitter is a function of the geographical setup with respect to the receiver, and also more importantly, the receiver sensitivity. For the measurement setup used thus far, the distance $(d)$ between the transmitter and receiver was low in proportion to their heights, thereby minimizing the effects of the ground. However, over longer ranges ( $\sim 8 \mathrm{~m}$ for the measurement setup used), the values of $d, d_{t}$, and $d_{r}$ in (3) would increase the maximum Fresnel zone radius $r$ with respect to the placement heights of the transmitter $\left(h_{t}\right)$ and receiver $\left(h_{r}\right)$. Due to this, the two-ray beam tracing model was used to compute longer range distances of the transmitter since it better accounted for received signals that had bounced off the ground [26]. For long distances with respect to the placement heights of the transmitter $\left(h_{t}\right)$ and receiver $\left(h_{r}\right)$, the range of the transmitter $(d)$ was determined using (5) as follows:

$$
d=\left(\frac{\operatorname{EIRP} \cdot G_{r}\left(h_{t} h_{r}\right)^{2}}{P_{r}}\right)^{\frac{1}{4}} .
$$

In (5), $P_{r}$ is the lowest power level detectable by a receiver, which is also known as the sensitivity of the receiver. A search for commercial transceivers that received ASK modulated signals at data rates of $5-10 \mathrm{~kb} / \mathrm{s}$, which are used for RFIDs and low power wireless sensing applications revealed sensitivities ranging from -65 to $-111 \mathrm{dBm}$ [29], [30]. Based on the receiver sensitivities found, for an omnidirectional antenna on the receiver side $\left(G_{r}=0 \mathrm{~dB}\right)$, the dipole-based module would be expected to have a minimum range between 25.32 and $357.66 \mathrm{~m}$ using (5). In free space, this range would be between 15-2995 m for receivers with sensitivities ranging from -65 to $-111 \mathrm{dBm}$, respectively.

In order to verify the correct operation of the dipole-based wireless sensor module, it was triggered to operate in the SENSING mode. The ASK modulated sensor information sent out by the module at different temperatures that was measured by the RTSA is shown in Fig. 24. The transmitted sensor data shows good agreement with the measurements carried out with the digital IR thermometer. The digital IR thermometer has an accuracy of $\pm 2.5^{\circ} \mathrm{C}$. [31]. 


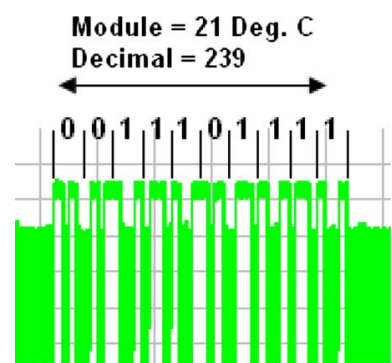

Digital IR $=22.6$ Deg. C

Fig. 24. ASK modulated temperature sensor data captured by the RTSA at room temperature (power versus time). Module: sensed temperature transmitted from module and captured by RTSA. Digital IR: temperature measured by the digital IR thermometer.

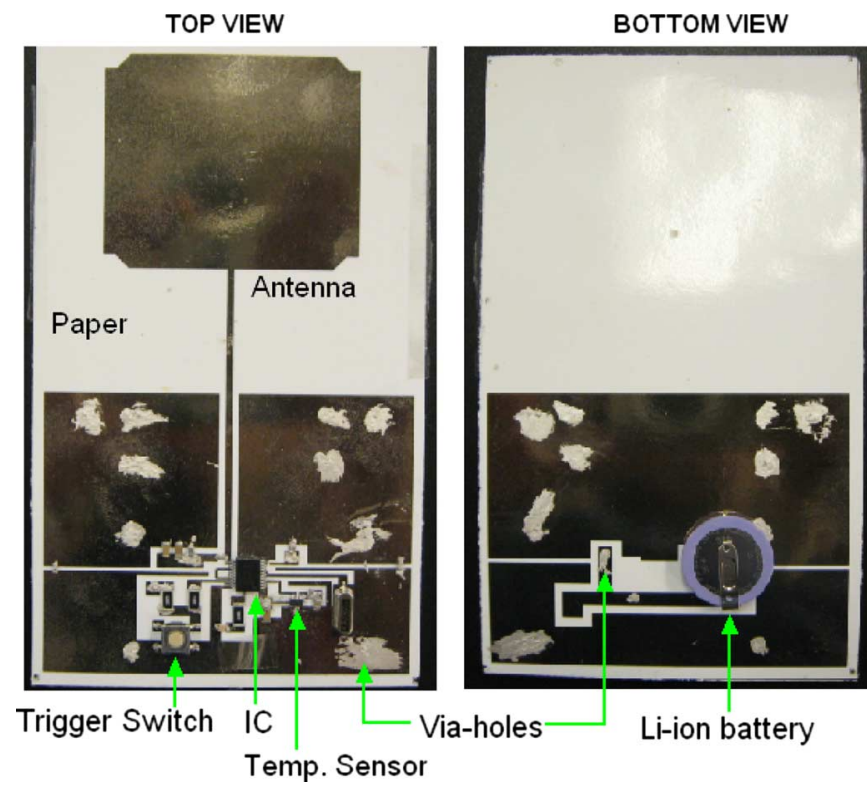

Fig. 25. Monopole-based wireless sensor module (two layer).

\section{B. Monopole-Based Wireless Sensor Module}

The assembled monopole-based wireless sensor module can be seen in Fig. 25. The filled via-holes can be seen as smudges on the ground plane in Fig. 25. Wireless link measurements were carried out with the monopole-based wireless sensor module by placing them at different temperatures. The measurement setup was similar to the one used for the dipole-based modules. The transmitter and receive antenna were placed at heights of 0.86 $\left(h_{t}\right)$ and $0.97\left(h_{r}\right) \mathrm{m}$, respectively, at $1.83 \mathrm{~m}(d)$ apart due to limitations with the measurement setup with the oven and refrigerator. The transmitted signal measured by the RTSA can be seen in Fig. 26, and was observed to be $-26 \mathrm{dBm}$ at a frequency of 904.4 MHz.

A wireless link budget similar to the one used for the dipole-based module was also carried out for the monopole-based module. The radius of the first Fresnel zone for the measurement setup used was determined to be $0.55 \mathrm{~m}$, which was well cleared by the placement heights. Using the free-space loss model of (4), the EIRP transmitted from the monopole-based module was thereby determined to be $4.91 \mathrm{dBm}$.

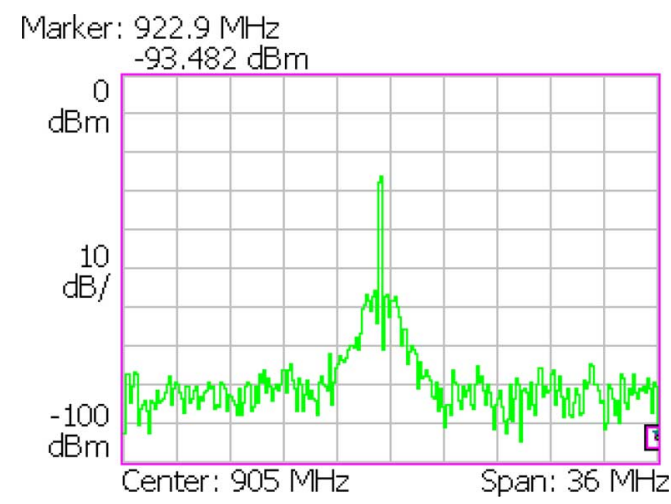

Fig. 26. RTSA measured ASK modulated signal for the monopole-based module (power versus frequency).

The minimum range $(d)$ of the monopole-based wireless sensor modules using the two-ray beam tracing model were determined to be between 51.1-721.6 $\mathrm{m}$ using (5) for receivers with sensitivities from -65 to $-111 \mathrm{dBm}$, respectively, and an omnidirectional antenna $\left(G_{r}=0 \mathrm{~dB}\right)$, respectively. However, in free space, the maximum range $(d)$ of the wireless sensor modules would increase to 82.66 on the lower end using (4) for a receiver sensitivities of $-65 \mathrm{dBm}$. However, for longer distances, the free-space model cannot be considered to be accurate since effects of the radius of the earth's curvature and environment need to be considered for distances over $10000 \mathrm{~m} \mathrm{[26].}$

In order to verify the correct operation of the monopole-based wireless sensor module, it was placed at different temperatures while triggered to operate in the SENSING mode. The ASK modulated sensor information sent out by the module at different temperatures that was measured by the RTSA is shown in Fig. 27. The transmitted sensor data shows good agreement with the measurements carried out with the digital IR thermometer. The digital IR thermometer has an accuracy of $\pm 2.5^{\circ} \mathrm{C}$ [31].

A comparison between the dipole- and monopole-based sensor modules can be seen in Table III. Efforts to ensure an impedance match between the dipole and the RF PA with the circuit close to its feed, and reduce RF leakage from the RF to the non-RF digital side of the circuit did improve the performance of the dipole-based module. In addition, the use of a low-precision high-volume cartridge to increase conductivity of the antenna structure also helped in increasing the amount of power being transmitter by over $20 \mathrm{~dB}$ from its predecessor in [12], as shown in Figs. 21 and 22. While the simulations revealed a directivity of $1.54 \mathrm{~dB}$, a more thorough analysis needs to be performed to study the effects of the printing process and the module circuit on the gain of the dipole antenna since the EIRP of the sensor module was determined to be only $-9.9 \mathrm{dBm}$.

By comparison, the monopole through the use of its grounded structure offered a significantly convenient way of integrating the antenna with the module circuitry with a better match and isolation. The planar square shape of the monopole also increased the area of the overlap between adjacent printed layers that could potentially decrease losses in the radiating structure and increase the gain. This can be seen with the significantly 


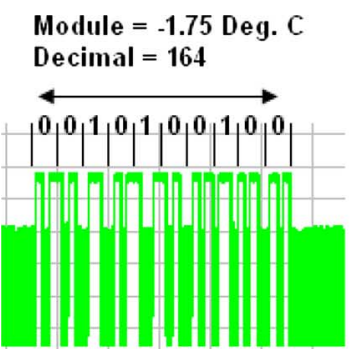

Digital IR $=-2$ Deg. $C$

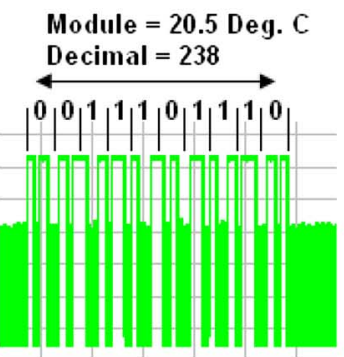

Digital IR $=19.8$ Deg. $C$

Module $=33.86$ Deg. $\mathrm{C}$ Decimal $=285$

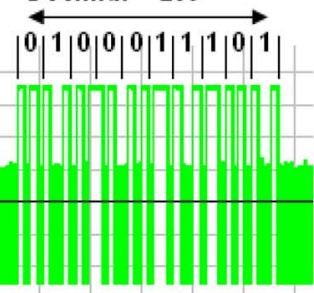

Digital IR $=34$ Deg. $C$

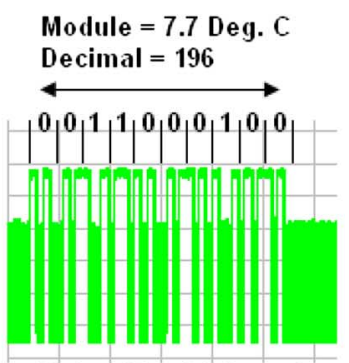

Digital IR $=6.9$ Deg. $C$

Module $=28.85$ Deg. $\mathrm{C}$ Decimal $=268$

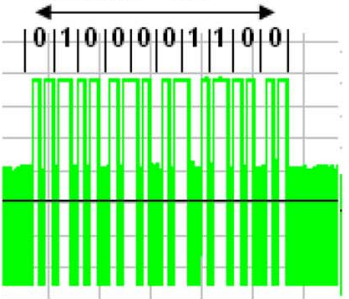

Digital IR $=28$ Deg. C

Module $=44.15$ Deg. $\mathrm{C}$ Decimal $=320$
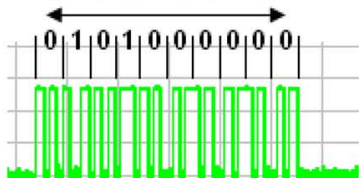

Hin

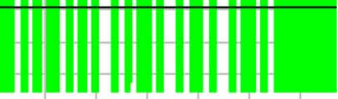

Digital IR $=$ 42.4 Deg. $C$
Fig. 27. ASK modulated temperature sensor data captured by the RTSA from $-2{ }^{\circ} \mathrm{C}$ to $42.4^{\circ} \mathrm{C}$ (power versus time). Module: sensed temperature transmitted from module and captured by RTSA. Digital IR: temperature measured by the digital IR thermometer.

TABLE III

PERformance COMPARISON BETWEen THE DiPOLEAND MONOPOLE-BASED SENSOR MODULES

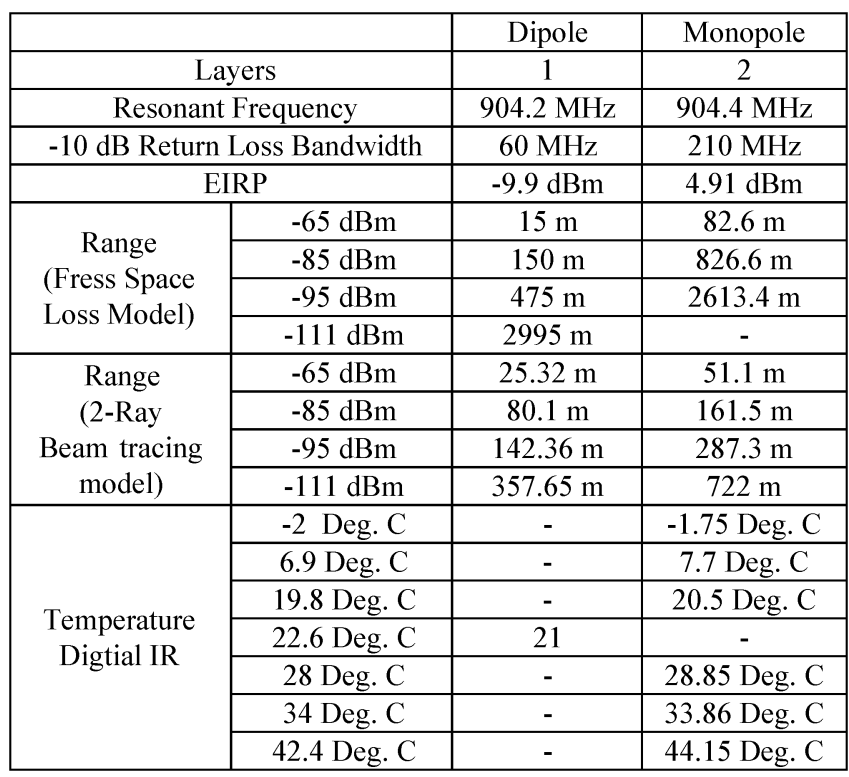

higher EIRP (4.91 dBm) given out, and correspondingly, the longer range of the monopole-based sensor module.
In addition, the monopole-based structure also offered more wideband performance compared the dipole-based modules, as can be seen from their respective return losses in Figs. 10 and 13. The $-10-\mathrm{dB}$ return loss of the monopole covers all three frequency bands, 860, 904.4, and $925 \mathrm{MHz}$ at which the RF PA in the transmitter could optimally operate. The dipole bandwidth was limited to only frequencies at 904.4 and $925 \mathrm{MHz}$.

While the size of the monopole and dipole radiating structure were comparable, the input feed of the monopole did significantly increase the overall size of the monopole-based sensor module. A shorter feed with a matching network could be used to decrease the dimension of the monopole-based module.

In addition, paper also offers excellent characteristics as a packaging material for sensors, especially temperature sensor considering its low thermal conductivity. The sensor measurements for both the dipole- and monopole-based sensor modules showed good agreement with digital IR thermometer that was used as a reference as was verified by its wireless link measurements.

\section{CONCLUSION}

Two different wireless sensor modules have been implemented on a paper substrate using inket-printing technology for the first time, and tested for wireless performance. The complete process including the system and antenna design, circuit layout, printing methodology, and assembly techniques that would potentially result in enhanced performance of the wireless sensor modules on paper was outlined. Wireless link measurements carried out on the complete prototypes on paper show acceptable performance on the communication and sensing side highlighting the feasibility of paper as a low-cost suitable eco-friendly packaging material as an active RFID-enabled wireless sensing platforms operating in the UHF range. Improved performance combined with the inherent cost and environmental advantages of paper over other substrates make paper-based wireless sensor modules an ideal choice for implementing large-scale RFID enables wireless sensor networks.

\section{ACKNOWLEDGMENT}

The authors would like to thank K. Rutkowski, SATIMO, Kennesaw, GA, for performing antenna radiation pattern measurements.

\section{REFERENCES}

[1] L. Yang and M. M. Tentzeris, "Design and characterization of novel paper-based inkjet-printed RFID and microwave structures for telecommunication and sensing applications," in IEEE MTT-S Int. Microw. Symp. Dig., Jun. 2007, pp. 1633-1636.

[2] L. Yang, A. Rida, R. Vyas, and M. M. Tentzeris, "RFID tag and RF structures on paper substrates using inkjet-printing technology," IEEE Trans. Microw. Theory Tech., vol. 55, no. 12, pt. 2, pp. 2894-2901, Dec. 2007.

[3] A. Rida, L. Yang, R. Vyas, S. Bhattacharya, and M. Tentzeris, "Design and integration of inkjet-printed paper-based UHF components for RFID and ubiquitous sensing applications," in IEEE Eur. Microw. Conf., Oct. 2007, pp. 724-727.

[4] A. Rida, L. Yang, R. Vyas, S. Basat, S. Bhattacharya, and M. Tentzeris, "Novel manufacturing processes for ultra-low-cost paper-based RFID tags with enhanced "wireless intelligence'," in IEEE Electron. Compon., Technol. Conf., May-Jun. 2007, pp. 773-776. 
[5] "EPC radio-frequency identity protocols class-1 generation-2 UHF RFID air interface version 1.0.9," EPCglobal, Lawrenceville, NJ, 2005.

[6] R. Vyas, A. Rida, L. Yang, and M. Tentzeris, "Design and development of a novel paper-based inkjet-printed RFID-enabled UHF (433.9 MHz) sensor node," in IEEE Asia-Pacific Microw. Conf., Dec. 2007, pp. 1-4.

[7] "Panasonic magnesium lithium coin batteries specifications," Panasonic, Osaka, Japan, 2005.

[8] "UHF Gen-2 system overview," Texas Instrum. Incorporated, Dallas, TX, Sep. 2005.

[9] U. Rohde, Microwave and Wireless Synthesizers: Theory and Design. Paterson, NJ: Wiley, 1997, pp. 1-5.

[10] J. Peatman, Embedded Design with the PIC18F452 Microcontroller. Upper Saddle River, NJ: Pearson, 2003, pp. 51-68, 116-131.

[11] S. Cripps, RF Power Amplifiers for Wireless Communication. Norwood, MA: Artech House, 1999, pp. 1-32.

[12] R. Vyas, A. Rida, L. Yang, and M. M. Tentzeris, "Design, integration and characterization of a novel paper based wireless sensor module," in IEEE MTT-S Int. Microw. Symp. Dig., Jun. 2008, pp. 1305-1308.

[13] L. Yang, S. Basat, and A. Rida, "Design and development of novel miniaturized UHF RFID tags on ultra-low-cost paper-based substrates," in IEEE Asia-Pacific Microw. Conf., Dec. 2006, pp. 1493-1496.

[14] L. Yang, A. Rida, T. Wu, S. Basat, and M. Tentzeris, "Integration of sensors and inkjet-printed RFID tags on paper-based substrates for UHF 'Cognitive Intelligence' applications,' in IEEE AP-S Int. Symp., Jun. 2007, pp. 1193-1196.

[15] C. Balanis, Antenna Theory. New York: Wiley, 1997, pp. 32-34, 133-143, 162, 412-414.

[16] A. Rida, L. Yang, and M. Tentzeris, "Design and characterization of novel paper-based inkjet-printed UHF antennas for RFID and sensing applications," in IEEE AP-S Int. Symp., Jun. 2007, pp. 2749-2752.

[17] M. Tentzeris, L. Yang, A. Rida, A. Traille, R. Vyas, and T. Wu, "Inkjetprinted RFID tags on paper-based substrates for UHF "cognitive intelligence" applications," in IEEE Pers. Indoor Mobile Radio Commun. Int. Symp., Sep. 2007, pp. 1-4.

[18] M. Tentzeris, L. Yang, A. Rida, A. Traille, R. Vyas, and T. Wu, "RFID's on paper using inkjet-printing technology: Is it the first step for UHF ubiquitous "cognitive intelligence" and "global tracking"?," in RFID Eurasia Conf., Sep. 2007, pp. 1-4.

[19] A. Rida, R. Vyas, S. Basat, A. Ferrer-Vidal, L. Yang, S. Bhattacharya, and M. Tentzeris, "Paper-based ultra-low-cost integrated RFID tags for sensing and tracking applications," in IEEE Electron. Compon. Technol. Conf., May-Jun. 2007, pp. 1977-1980.

[20] B. Kim, S. Nikolaou, G. E. Ponchak, Y.-S. Kim, J. Papapolymerou, and M. M. Tentzeris, "A curvature CPW-fed ultra-wideband monopole antenna on liquid crystal polymer substrate using flexible characteristics," in IEEE AP-S Int. Symp., Albuquerque, NM, Jul. 2006, pp. 1667-1670.

[21] S. H. Lee, J. K. Park, and J. N. Lee, "BA novel CPW-fed ultra-wideband antenna design," Microw. Opt. Technol. Lett., vol. 44, no. 5, pp. 393-396, Mar. 2005.

[22] A. Rida, L. Yang, R. Vyas, D. Staiculescu, and M. M. Tentzeris, "Novel miniaturized inkjet-printed paper-based UHF components for RFID and sensing applications," J. Eur. Microw. Assoc. (Special Issue), vol. 3, no. 4, pp. 336-340, Dec. 2007.

[23] H. W. Ott, Noise Reductaion Techniques in Electronic Syatems, 2nd ed. New York: Wiley, 1988, pp. 73-115.

[24] M. Carter, J. Colvin, and J. Sears, "Characterization of conductive inks deposited with maskless mesoscale material deposition," in TMS2006, San Antonio, TX, Mar. 12-16, 2006. [Online]. Available: http://www. nanoscale.com/markets/

[25] M. Tentzeris, "Novel paper-based inkjet printed antennas and wireless sensor modules," in IEEE COMCAS, May 2008, pp. 1-8.

[26] J. Seybold, Introduction to RF Propagation. Hoboken, NJ: Wiley, 2005, pp. 111-133, 163-179.

[27] T. Kvaksrud, "Range measurements in an open field environment," Texas Instrum. Incorporated, Dallas, TX, Design Note DN018, 2008.

[28] "Motorola RFID antennas for fixed readers," Motorola, Motorola AN-400 RFID Antenna Data Sheet, 2007.

[29] "UHF ASK/FSK transceiver: ATA5429," Atmel, San Jose, CA, Data Sheet, Oct. 2007.

[30] P. V. Nikitin and K. V. S. Rao, "Performance limitations of passive UHF RFID systems," in IEEE AP-S Int. Symp., Jul. 2006, pp. 1011-1014.

[31] “Radioshack Digital Infrared Thermometer Owner's Manual," Radioshack Corporation, Fort Worth, TX, 2001.

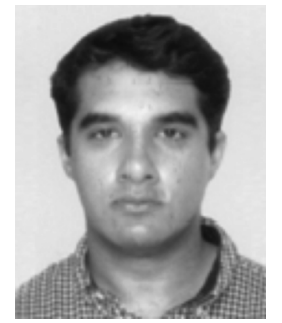

Rushi Vyas (S'07) received the B.S. degree in electrical engineering (with a background in $\mathrm{RF}$, analog circuit design and embedded systems) from the Georgia Institute of Technology, Atlanta, in 2005 , and is currently working toward the Ph.D degree in electrical and computer engineering at the Georgia Institute of Technology.

He is currently a Graduate Research Assistant with the Georgia Electronic Design Center, Atlanta, GA. His research has concerned areas of antenna design in the millimeter-wave and UHF range, PA design and characterization, and its integration primarily on eco-friendly organic substrates using inket-printing techniques for automotive radar and RFID applications. His current research involves combining wireless, embedded systems, and power electronics techniques in the design and prototyping of enhanced range, power scavenging tags for RFID and remote sensing applications.

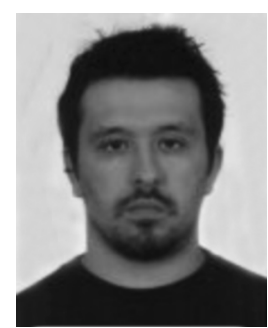

Vasileios Lakafosis (S'07) received the Diploma degree in electrical and computer engineering from the National Technical University, Athens, Greece, in 2006, and is currently working toward the Ph.D. degree in electrical and computer engineering at the Georgia Institute of Technology, Atlanta.

From 2006 to 2007, he was an Electrical and Computer Engineer with Intracom Telecom, Athens, Greece. In 2007, he joined the School of Electrical and Computer Engineering, Georgia Institute of Technology. He is currently a Research Assistant with the ATHENA Group, Georgia Electronic Design Center, Atlanta, GA. His current research is focused on communication protocols in the area of wireless ad hoc, sensor, and mesh networks.

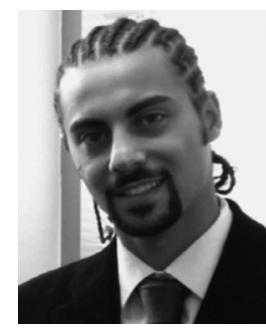

Amin Rida (S'06) received the B.S. degree in electrical engineering from the Georgia Institute of Technology, Atlanta, in 2006, and is currently working toward the Ph.D. degree at the Georgia Institute of Technology.

$\mathrm{He}$ is currently with the Georgia Electronic Design Center (GEDC), Atlanta, GA. His research interests include characterization of organic substrates for RF applications, design of UHF antennas for RFID applications, and development of wireless transceivers for sensing and power scavenging applications.

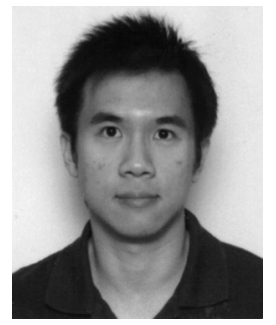

Napol Chaisilwattana received the B.S. degree in electrical engineering from the University of Minnesota, Twin Cities, in 2006 and the M.S. degree in electrical engineering from the Georgia Institute of Technology, Atlanta, in 2008.

During his graduate studies, he was a Graduate Research Assistant with the ATHENA Group, Georgia Electronic Design Center, Atlanta, GA. His research included finite-difference time-domain (FDTD) modeling for biomedical impedance cardiograph applications and antenna design for sensor nodes. Prior to graduate school, he was an Intern with $3 \mathrm{M}$, where he conducted studies revolving around metal mount tags. His current interests revolve around RFID, antenna design, and EM modeling.

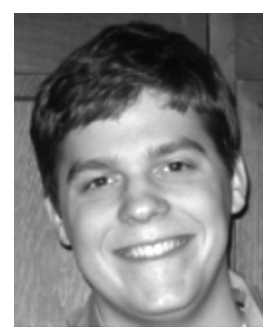

Scott Travis received the B.S. degree in electrical engineering (with highest honors) from the Georgia Institute of Technology, Atlanta, in 2008.

He was an Undergraduate Research Assistant with the ATHENA Group, Georgia Electronic Design Center, Atlanta, GA, during his junior and senior years of study. His research concerns EM theory, high-frequency design, and radar technologies. 


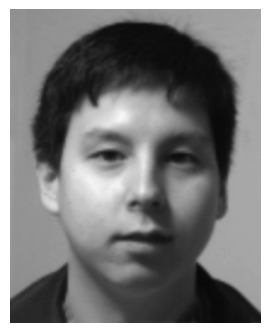

Jonathan Pan received the Bachelors degree in electrical engineering from the Georgia Institute of Technology, Atlanta, in 2008, and is currently working toward the Ph.D. degree in electrical engineering at the Georgia Institute of Technology.

Since 2008, he has been with the ATHENA Group, Georgia Electronic Design Center, Atlanta, GA During this time, his research has included printable RFID, antenna design, and most recently, design of microwave filters.

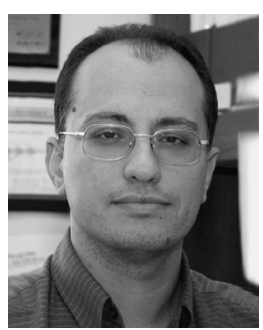

Manos M. Tentzeris (S'89-M'92-SM'03) received the Diploma degree in electrical and computer engineering (magna cum laude) from the National Technical University of Athens, Athens, Greece, and the M.S. and Ph.D. degrees in electrical engineering and computer science from The University of Michigan at Ann Arbor.

$\mathrm{He}$ is currently a Professor with the School of Electrical and Computer Engineering, Georgia Institute of Technology, Atlanta. He has helped develop academic programs in highly integrated/multilayer packaging for RF and wireless applications using ceramic and organic flexible materials, paper-based RFIDs and sensors, microwave microelectromechanical systems (MEMs), system-on-package (SOP)-integrated (ultra-wideband (UWB), mutliband, conformal) antennas, and adaptive numerical electromagnetics (finite difference time domain (FDTD) and multiresolution algorithms). He heads the ATHENA Group, Georgia Electronic Design Center, Atlanta, GA (20 researchers). He is the Georgia Electronic Design Center Associate Director for RFID/sensors research. From 2003 to 2006, he was the Georgia Institute of Technology NSF-Packaging Research Center Associate Director for RF Research and the RF Alliance Leader. In Summer 2002, he was a Visiting Professor with the Technical University of Munich, Munich, Germany, where he introduced a course in the area of high-frequency packaging. He has given more than 50 invited talks to various universities and companies in
Europe, Asia, and America. He has authored or coauthored over 320 papers in refereed journals and conference proceedings, three books, and 17 book chapters. He is an Associate Editor for the International Journal on Antennas and Propagation.

Dr. Tentzeris is a member of URSI-Commission D and the MTT-15 committee. He is an Associate Member of the European Microwave Association (EuMA). He is a Fellow of the Electromagnetic Academy and a member of the Technical Chamber of Greece. He was the Technical Program Committee (TPC) chair for the 2008 IEEE Microwave Theory and Techniques Society (IEEE MTT-S) International Microwave Symposium (IMS) and chair of the 2005 IEEE Computational Electromagnetics-Time Domain (CEM-TD) Workshop. He is the vice-chair of the RF Technical Committee (TC16) of the IEEE Components, Packaging, and Manufacturing Technology (CPMT) Society. He is the founder and chair of the RFID Technical Committee (TC24) of the IEEE MTT-S and the secretary/treasurer of the IEEE C-RFID. He has organized various sessions and workshops on RF/wireless packaging and integration, RFIDs, and numerical techniques/wavelets in IEEE Electronic Components Technology Conference (ECTC), IMS, Vehicular Technology Conference (VTC), and Antennas and Propagation Society (AP-S) symposia, all of which he is a member of the TPC in the area of components and RF. He is an associate editor of the IEEE TRANSACTIONS ON MICROWAVE THEORY AND TECHNIQUES and the IEEE TRANSACTIONS ON ADVANCED PACKAGING. He was the recipient/corecipient of the 2007 IEEE AP-S Symposium Best Student Paper Award, the 2007 IEEE MTT-S IMS Third Best Student Paper Award, the 2007 International Symposium on Antenna Propagation (ISAP) 2007 Poster Presentation Award, the 2006 IEEE MTT-S Outstanding Young Engineer Award, the 2006 Asia-Pacific Microwave Conference Award, the 2004 IEEE TRANSACTIONS ON AdVANCED PACKAGING Commendable Paper Award, the 2003 National Aeronautics and Space Administration (NASA) Godfrey Art Anzic Collaborative Distinguished Publication Award, the 2003 IBC International Educator of the Year Award, the 2003 IEEE CPMT Outstanding Young Engineer Award, the 2002 International Conference on Microwave and Millimeter-Wave Technology Best Paper Award (Beijing, China), the 2002 Georgia Tech Electrical and Computer Engineering (ECE) Outstanding Junior Faculty Award, the 2001 Applied Computational Electromagnetics (ACES) Conference Best Paper Award and the 2000 National Science Foundation (NSF) CAREER Award, and the 1997 Best Paper Award of the International Hybrid Microelectronics and Packaging Society. 